УДК 621.355

\title{
АЛГОРИТМ ПРОВЕДЕНИЯ ИСПЫТАНИЙ ДЛЯ ОПРЕДЕЛЕНИЯ ПАРАМЕТРОВ ОТКЛИКА СИСТЕМЫ НАКОПЛЕНИЯ ЭЛЕКТРИЧЕСКОЙ ЭНЕРГИИ
}

\author{
Андрианова Людмила Прокопьевна \\ д-р техн. наук, профессор \\ Павлова Зухра Хасановна \\ д-р техн. наук, профессор \\ Рябишина Лилия Амировна \\ канд. техн. наук, доцент \\ Хакимьянов Марат Ильгизович \\ д-р техн. наук, профессор \\ Хазиева Регина Тагировна \\ канд. техн. наук, доцент \\ ФГБОУ ВО Уфимский государственный \\ нефтяной технический университет
}

\begin{abstract}
Аннотация: В статье дана краткая характеристика основных и дополнительных параметров СНЭЭ, установленных для испытаний действующими нормативными документами. Представлена обобщенная архитектура СНЭЭ, включающая подсистемы: основную и вспомогательную, контроля и управления; распределения и подключения с указанием точек подключения к сети помощью основного и вспомогательного стыковочных выводов. Изложена методика определения и испытаний параметров отклика: время отклика и скорость изменения выходной переменной СНЭЭ на входное ступенчатое воздействие; алгоритм и этапы испытаний параметров отклика.

Ключевые слова: Системы накопления электрической энергии, параметры и характеристики; типовая архитектура, точка подключения к сети; отклик системы на единичное ступенчатое воздействие; этапы испытаний.
\end{abstract}




\title{
TEST ALGORITHM FOR DETERMINING THE RESPONSE PARAMETERS OF THE ELECTRIC ENERGY STORAGE SYSTEM
}

\author{
Andrianova Lyudmila Prokofievna \\ Pavlova Zuhra Khasanovna \\ Ryabishina Liliya Amirovna \\ Khakimjanov Marat Ilgizovich \\ Khazieva Regina Tagirovna
}

\begin{abstract}
The article provides a brief description of the main and additional parameters of SNEE, established for testing by current regulatory documents. A generalized SNEE architecture is presented, including subsystems: main and auxiliary, control and control; distribution and connection with the indication of connection points to the network using the main and auxiliary connecting pins. The method of determining and testing the response parameters is described: response time and rate of change of the output variable of the SNEE to the input step effect; algorithm and stages of testing the response parameters.
\end{abstract}

Key words: Electrical energy storage systems, parameters and characteristics; typical architecture, connection point to the network; system response to a single step impact; test stages.

\section{Введение.}

В последние десятилетия в связи с интенсивным развитием технологий накопления энергии и созданием накопителей электрической энергии $\mathrm{c}$ высокими энергетическими показателями (мощности, энергоемкости, КПД и быстродействие), переосмыслена их место и роль в современных энергосистемах [1].

Накопители энергии в составе энергосистемы позволяют поддерживать баланс активной мощности, участвуя в энергообмене с энергосистемой, регулируя в течение суток выработку и потребление электроэнергии, причем они могут быть установлены в любой точке энергосистемы, там, где в них есть потребность. Кроме управления активной мощностью они одновременно способны выполнять функции регулирования напряжения в точке подключения, функции активного фильтра высших гармоник напряжения и тока, а также решать проблему симметрирования трехфазной электрической сети $[3,4]$. 
Для СНЭЭ, предназначенных для работы в составе Единой энергетической системы России и технологически изолированных территориальных электроэнергетических систем и относящихся к объектам диспетчеризации, установлены основные и дополнительные параметры, подлежащие испытаниям в соответствии с нормативными документами $[2,3,4]$.

\section{Параметры СНЭЭ}

Перечень параметров СНЭЭ, установленных для испытаний их величин в соответствии с $[2,3,4]$ представлен в таблице 1 .

\section{Таблица 1}

\section{Параметры СНЭЭ [5, 6]}

\begin{tabular}{|c|c|}
\hline Наименование параметра & Краткая характеристика \\
\hline $\begin{array}{l}\text { 1. Номинальная энергоемкость, } \\
\text { Вт·ч }\end{array}$ & $\begin{array}{l}\text { Номинальная энергоемкость - энергия, которую СНЭЭ } \\
\text { может отдать в ТПН в условиях проведения испытаний. }\end{array}$ \\
\hline $\begin{array}{l}\text { 2. Входная и выходная мощности, } \\
\text { Вт, вар, В·А }\end{array}$ & $\begin{array}{l}\text { Мощности, которые СНЭЭ может поглощать или } \\
\text { отдавать в течение определенного времени в ТПН в } \\
\text { условиях эксплуатации }\end{array}$ \\
\hline 3. Эффективность заряда-разряда, \% & $\begin{array}{l}\text { Эффективность заряда-разряда СНЭЭ - это отношение } \\
\text { полной выходной энергии к полной входной энергии в } \\
\text { течение одного цикла заряда-разряда системы при } \\
\text { нормированных входной и выходной мощностях }\end{array}$ \\
\hline $\begin{array}{l}\text { 4. Расчетный срок службы (годы, } \\
\text { рабочие циклы) }\end{array}$ & $\begin{array}{l}\text { Расчетный срок службы СНЭЭ - момент времени, в } \\
\text { который происходит ухудшение любого из параметров: } \\
\text { фактической энергоемкости, входной и выходной } \\
\text { мощности, отклика системы до уровней, установленных } \\
\text { в технических условиях конкретной СНЭЭ в качестве } \\
\text { значения показателей в конце срока службы }\end{array}$ \\
\hline 5. Отклик системы: & $\begin{array}{l}\text { - время отклика на единичное ступенчатое возмущение, } \\
\text { с; } \\
\text { - скорость изменения выходной переменной, Вт/с); }\end{array}$ \\
\hline $\begin{array}{l}\text { 6. Мощность потребления } \\
\text { вспомогательной подсистемы, Вт }\end{array}$ & $\begin{array}{l}\text { Мощность потребления вспомогательной подсистемы } \\
\text { СНЭЭ - это мощность, необходимая для работы } \\
\text { вспомогательной подсистемы }\end{array}$ \\
\hline 7. Саморазряд, Вт·ч/ч & $\begin{array}{l}\text { Саморазряд СНЭЭ - это потери энергии системы в } \\
\text { остановленном состоянии за стандартный период } \\
\text { времени. }\end{array}$ \\
\hline $\begin{array}{l}\text { 8. Нормированный диапазон } \\
\text { напряжений, В }\end{array}$ & $\begin{array}{l}\text { Нормированный диапазон напряжений СНЭЭ - это } \\
\text { диапазон значений напряжения в ТПН, в котором } \\
\text { система должна оставаться подключенной к сети. }\end{array}$ \\
\hline
\end{tabular}




\begin{tabular}{|l|l|}
\hline $\begin{array}{l}\text { 9. Нормированный диапазон частот, } \\
\text { Гц }\end{array}$ & $\begin{array}{l}\text { Нормированный диапазон частот СНЭЭ - диапазон } \\
\text { значений частоты в ТПН, в котором система должна } \\
\text { оставаться подключенной к сети. }\end{array}$ \\
\hline
\end{tabular}

Все параметры СНЭЭ, указанные в таблице 3, согласно ГОСТ [2, 3] измеряют или рассчитывают в точке подключения к сети (ТПН).

\section{Технические требования, предъявляемые к СНЭЭ}

В зависимости от решаемых задач к СНЭЭ предъявляются технические требования $[2,3,4]$ :

- по функциональности (покрытие пиковых нагрузок, обеспечение требуемой частоты, режим работы виртуальной синхронной машины и др.);

- по параметрам подсистемы накопления энергии (энергоемкость, мощность и др.);

- по параметрам подсистемы преобразования энергии (время отклика, контроль статизма, мощность, мощность короткого замыкания и др.);

- по параметрам подсистемы защиты.

Для разработки оптимального решения, повышения адаптивности и эффективности функционирования системы требования к СНЭЭ должны быть четко определены с учетом требований электрической сети. На этапе проектирования, на системном уровне и после уточнения задач должны быть определены требования к СНЭЭ путем задания требований к соответствующим рабочим характеристикам СНЭЭ, включая [4]:

- нормированную входную и выходную мощность;

- кратковременную входную и выходную мощность;

- нормированную энергоемкость;

- время отклика;

- энергопотребление вспомогательной подсистемой;

- саморазряд;

- эффективность заряда-разряда;

- эффективность заряда-разряда рабочего цикла;

- время восстановления;

- значение показателей рабочих характеристик в конце срока службы.

Расчетный срок службы СНЭЭ должен быть указан на этапе проектирования. Но по истечении срока службы параметры СНЭЭ могут не соответствовать значениям показателей в конце срока службы. 
Признаками этого могут служить:

- фактические значения входной и выходной энергоемкости СНЭЭ ниже значений, установленных для момента окончания срока службы;

- значения входной и выходной мощности во время процесса заряда и разряда системы ниже значений, определенных для момента окончания срока службы;

- эффективность заряда-разряда ниже величин, установленных для момента окончания срока службы;

- время отклика выше значений, определенных для момента окончания срока службы.

Требуемое время отклика устанавливается в соответствии с [4] для следующих режимов работы СНЭЭ:

- для автономной работы, режима следования за нагрузкой, работы по графику и дистанционного диспетчерского управления;

- для запуска СНЭЭ из режима ожидания;

- для переключения СНЭЭ из режима отбора активной мощности в режим отдачи активной мощности.

Для обеспечения устойчивости энергосистемы по запросу субъекта оперативно диспетчерского управления заказчик СНЭЭ должен предоставить информацию о значениях следующих параметров:

- время отклика;

- скорость изменения выходной переменной;

- токи подпитки точки короткого замыкания;

- способность поддержания напряжения в случае перенапряжения, прерывания или провала напряжения в сети;

- характеристика и способность регулирования.

Обобщенная архитектура СНЭЭ.

Типовая архитектура СНЭЭ со всеми структурными компонентами приведена на рис. 1. 


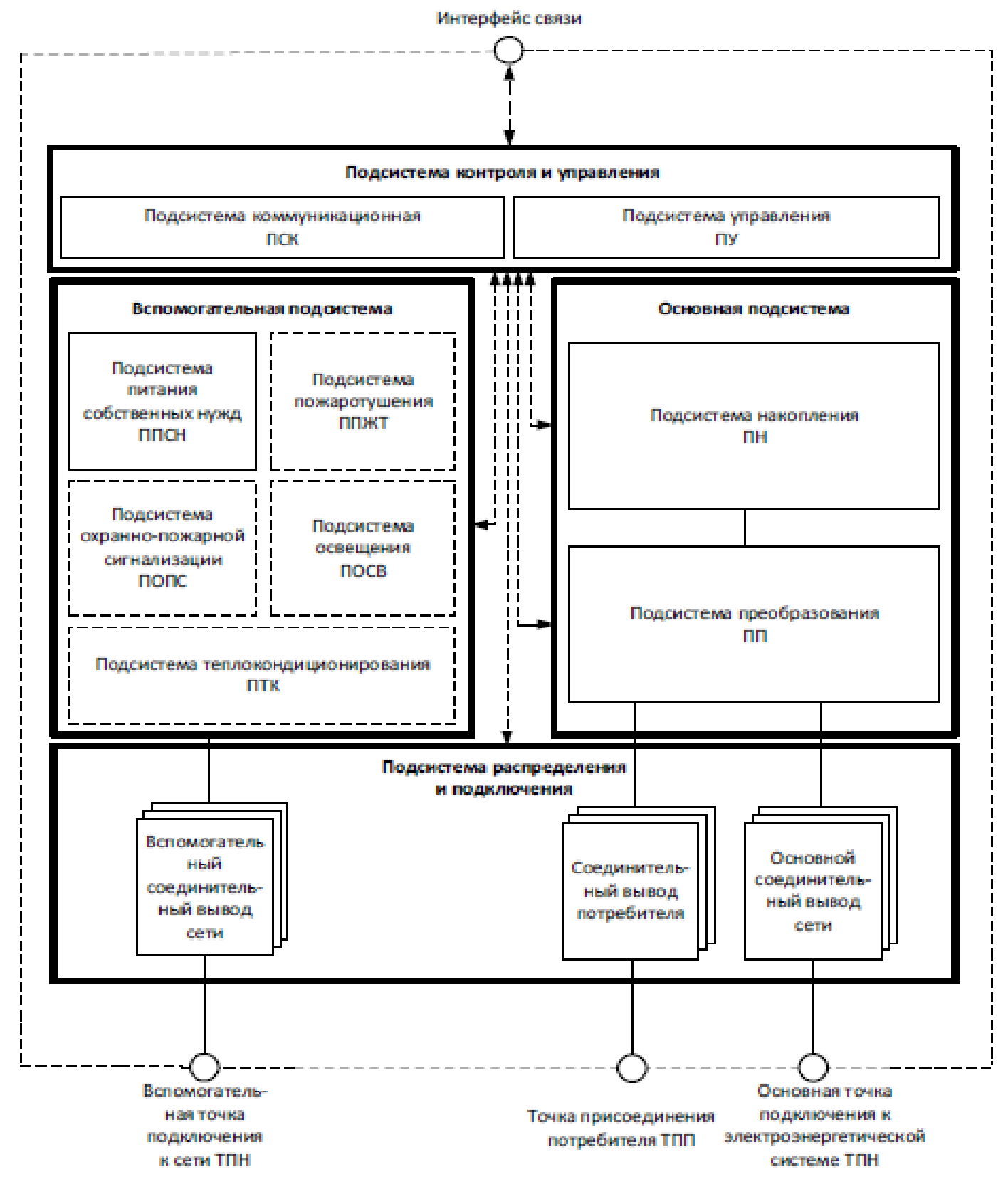

\section{Рис. 1. Обобщенная архитектура СНЭЭ}

\section{Методика определения и испытаний параметров отклика СНЭЭ}

Время отклика СНЭЭ на единичное ступенчатое возмущение - это период времени между моментом $T_{0}$, когда система, находящаяся в состоянии ожидания, получает уставку (или когда параметр энергосистемы изменяется достаточно для вызова отклика системы) и моментом $T_{3}$, когда активная мощность СНЭЭ в ТПН достигает значения в пределах 2\% от значения уставки (рис. 2) [3, с.17]. 
Типовой уставкой для определения времени отклика на единичное ступенчатое возмущение является значение нормированной входной или выходной мощности.

Скорость изменения выходной переменной СНЭЭ - средняя скорость изменения активной мощности $P\left(T_{2}\right)$ и $P\left(T_{1}\right)$ в единицу времени между моментами времени $T_{2}$ и $T_{1}$ (рис. 2 ), $R R, \mathrm{~B}$ /с, определяется по формуле

$$
R R=\frac{P\left(T_{2}\right)-P\left(T_{1}\right)}{T_{2}-T_{1}} \text {, где } T_{1} \text { - момент времени, в который активная мощность }
$$
в ТПН становится выше значения $10 \%$ уставки;

$T_{2}$ - момент времени, в который активная мощность в ТПН становится выше значения $90 \%$ уставки.

Типовыми уставками для определения скорости изменения выходной переменной являются нормированные входная и выходная мощности соответственно.

Время отклика СНЭЭ измеряют в соответствии с [3, с.15] по методике, изложенной ниже. СНЭЭ должна поглощать или отдавать активную мощность в соответствии с уставкой, настроенной на нормированную входную или выходную мощность. Уставку и входную или выходную мощность в ТПН фиксируют при помощи системы сбора данных через равные интервалы времени с требуемым временным разрешением.

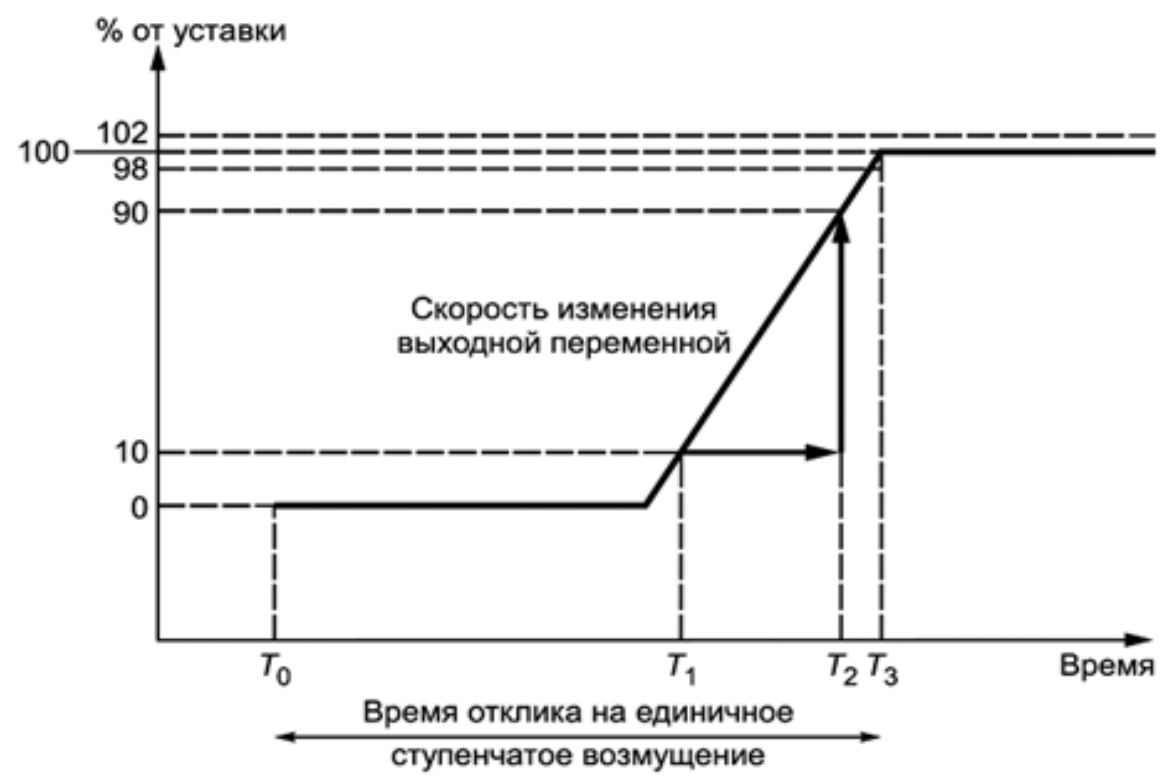

Рис. 2. Время отклика на единичное ступенчатое возмущение и скорость изменения выходной переменной СНЭЭ [3, с.16] 
Для определения отклика системы регистрируют в соответствии с рис. 3 четыре значения времени отклика на единичное ступенчатое возмущение ( $\left.S R T_{1}, S R T_{2}, S R T_{3}, S R T_{4}\right)$ и четыре скорости изменения выходной переменной ( $\left.R R_{1}, R R_{2}, R R_{3}, R R_{4}\right)$. Для задач обеспечения устойчивости энергосистемы при определении $T_{0 \mathrm{~b}}, T_{0 \mathrm{c}}, T_{0 \mathrm{~d}}$ И $T_{0 \mathrm{e}}$ учитывают дополнительное время между моментом регистрации параметра энергосистемы (частоты, напряжения) и моментом задания уставки системой контроля и управления СНЭЭ.

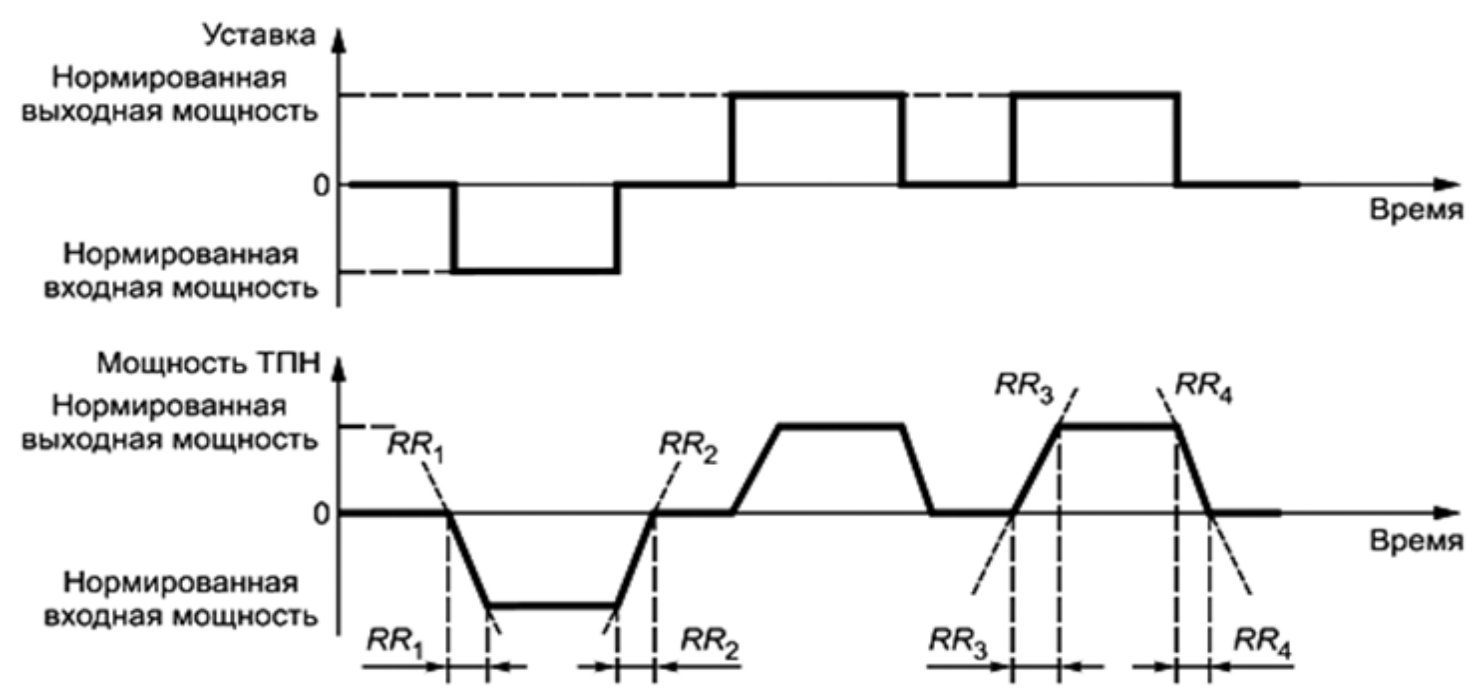

Рис. 3. Этапы определения отклика СНЭЭ [3, с.24]

\section{Алгоритм проведения испытаний}

Этап №1. Заряжают или разряжают СНЭЭ до степени заряженности $\mathrm{C} 3=50 \%$;

Этап №2. Задают уставку равной нулю. Уставку не меняют до тех пор, пока выходная мощность не составит $(0 \pm 2) \%$ от нормированной входной мощности.

Этап №3. Изменяют уставку на нормированную входную мощность. Уставку не меняют до тех пор, пока активная мощность в ТПН не достигнет уровня в пределах $2 \%$ от нормированной входной мощности (рис. 4).

Этап №4. Задают уставку равной нулю. Уставку не меняют до тех пор, пока активная мощность в ТПН не достигнет уровня $(0 \pm 2) \%$ от нормированной входной мощности (рис. 5). 

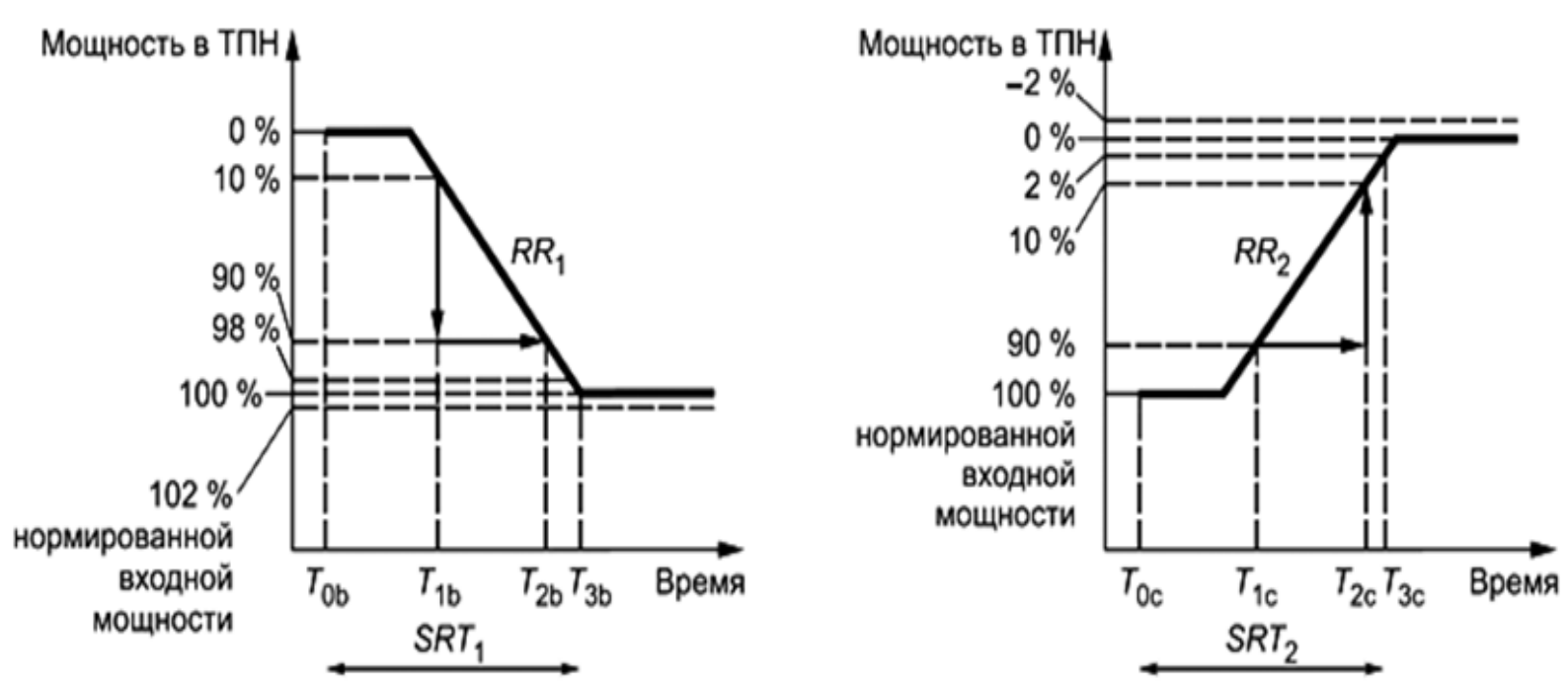

Рис. 4. Этап №3[3, с. 24]

Рис. 5. Этап №4[3, с. 24]

Пояснения к рис. 4 - Этап №3:

$T_{0 \mathrm{~b}}$ - момент времени, в который СНЭЭ получает значение уставки;

$T_{1 \mathrm{~b}}$ - момент времени, в который активная мощность в ТПН становится выше $10 \%$ от нормированной входной мощности;

$T_{2 \mathrm{~b}}$ - момент времени, в который активная мощность в ТПН становится выше 90\% от нормированной выходной мощности;

$T_{3 \mathrm{~b}}$ - момент времени, в который активная мощность в ТПН достигает уровня $\pm 2 \%$ от целевого значения нормированной выходной мощности;

$R R_{1}$ - скорость изменения выходной переменной - отношение разности значений активной мощности в моменты времени $T_{2 \mathrm{~b}}$ и $T_{1 \mathrm{~b}}$ К интервалу времени между моментами времени $T_{2 \mathrm{~b}}$ и $T_{1 \mathrm{~b}}$;

$S R T_{1}$ - время отклика на единичное ступенчатое возмущение - интервал времени между моментами времени $T_{3 \mathrm{~b}}$ и $T_{0 \mathrm{~b}}$.

\section{Пояснения к рис. 5 - Этап №4:}

$T_{1 \mathrm{c}}$ - момент времени, в который активная мощность в ТПН становится ниже 90\% от нормированной входной мощности;

$T_{2 \mathrm{c}}$ - момент времени, в который активная мощность в ТПН становится ниже $10 \%$ от нормированной входной мощности;

$T_{3 \mathrm{c}}$ - момент времени, в который активная мощность в ТПН достигает уровня $\pm 2 \%$ от целевого значения нормированной входной мощности. 
$R R_{2}$ - скорость изменения выходной переменной СНЭЭЭ - отношение разности значений активной мощности в моменты времени $T_{2 \mathrm{c}}$ И $T_{1 \mathrm{c}} \mathrm{K}$ интервалу времени между моментами времени $T_{2 \mathrm{c}}$ и $T_{1 \mathrm{c}}$;

$S R T_{2}$ - время отклика на единичное ступенчатое возмущение - интервал времени между моментами времени $T_{3 \mathrm{c}}$ и $T_{0 \mathrm{c}} . T_{0 \mathrm{c}}$ - момент времени, в который СНЭЭ получает значение уставки.

Этап №5. Заряжают или разряжают СНЭЭ до степени заряженности $\mathrm{C} 3=50 \%$ или до значения энергоемкости, согласованной между поставщиком и заказчиком СНЭЭ;

Этап №6. Изменяют уставку на нормированную выходную мощность. Уставку не меняют до тех пор, пока активная мощность в ТПН не достигнет уровня в пределах 2\% от нормированной выходной мощности (рис. 6).

Этап №7. Задают уставку равной нулю. Уставку не меняют до тех пор, пока активная мощность в ТПН не достигнет уровня $(0 \pm 2) \%$ от нормированной выходной мощности (рис. 7).
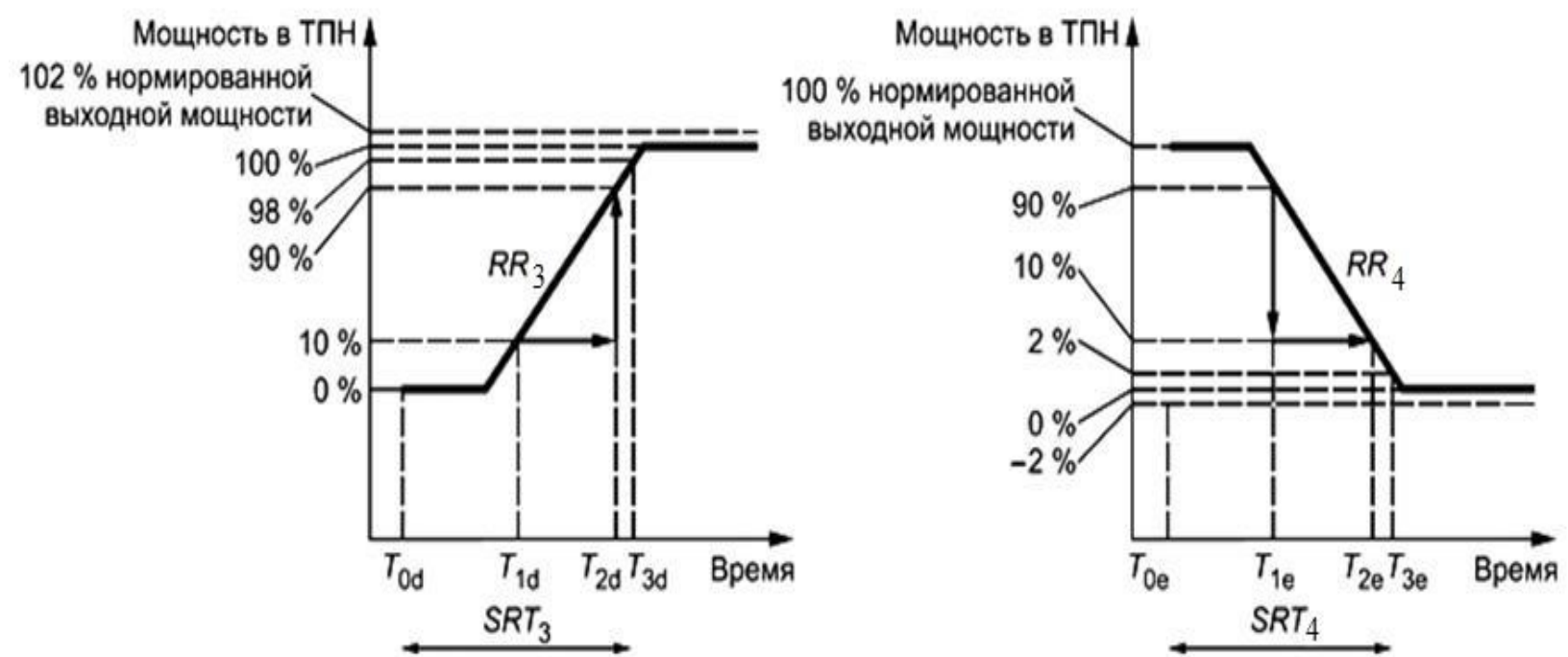

Рис. 6. Этап №6 [3, с. 24]

Рис. 7. Этап №7[3, с. 24]

Пояснения к рис. 6 - Этап №6:

$T_{0 \mathrm{~d}}$ - момент времени, в который СНЭЭ получает значение уставки;

$T_{1 \mathrm{~d}}$ - момент времени, в который активная мощность в ТПН становится выше $10 \%$ от нормированной выходной мощности; 


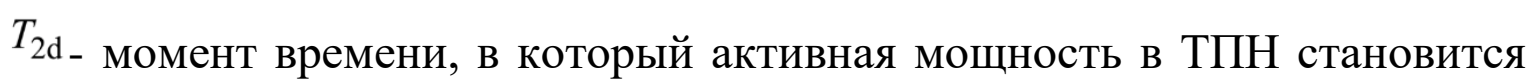
выше 90\% от нормированной выходной мощности;

$T_{3 \mathrm{~d}}$ - момент времени, в который активная мощность в ТПН достигает уровня $\pm 2 \%$ от целевого значения нормированной выходной мощности.

$R R_{3}$ - скорость изменения выходной переменной СНЭЭЭ - отношение разности значений активной мощности в моменты времени $T_{2 \mathrm{~d}}$ И $T_{1 \mathrm{~d}} \mathrm{~K}$ интервалу времени между моментами времени $T_{2 \mathrm{~d}}$ и $T_{1 \mathrm{~d}}$;

$S R T_{3}$ - время отклика на единичное ступенчатое возмущение - интервал времени между моментами времени $T_{3 \mathrm{~d}}$ И $T_{0 \mathrm{~d}}$.

Пояснения к рис. 7 - Этап №7:

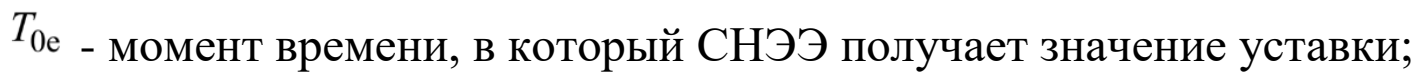

$T_{1 \mathrm{e}}$ - момент времени, в который активная мощность в ТПН становится ниже 90\% от нормированной выходной мощности;

$T_{2 \mathrm{e}}$ - момент времени, в который активная мощность в ТПН становится ниже $10 \%$ от нормированной выходной мощности;

$T_{3 \mathrm{e}}$ - момент времени, в который активная мощность в ТПН достигает уровня $\pm 2 \%$ от целевого значения нормированной выходной мощности.

$R R_{4}$ - скорость изменения выходной переменной СНЭЭ - отношение разности значений активной мощности в моменты времени $T_{2 \mathrm{e}}$ И $T_{1 \mathrm{e}} \mathrm{K}$ интервалу времени между моментами времени $T_{2 \mathrm{e}}$ и $T_{1 \mathrm{e}}$;

$S R T_{4}$ - время отклика на единичное ступенчатое возмущение - интервал времени между моментами времени $T_{3 \mathrm{e}}$ И $T_{0 \mathrm{e}}$.

Скорость сбора данных должна минимум в два раза превышать отношение полной нормированной мощности к скорости изменения выходной переменной системы.

Заключение. Параметры отклика (время отклика на единичное ступенчатое возмущение и скорость изменения выходной переменной СНЭЭ) относятся к параметрам системы, отвечающим за качество функционирования и за расчетный срок службы СНЭЭ. Так как с течением времени наблюдается тенденция ухудшения параметров СНЭЭ, и как следствие, уменьшение установленного на стадии проектирования расчетного срока службы. Для оценки соответствия фактического срока службы расчетному сроку службы, проводят испытания параметров СНЭЭ, включая и параметры отклика СНЭЭ. 
Методика измерения и испытания параметров отклика СНЭЭ описана на базе информации, приведенной в современных национальных стандартах Российской Федерации, гармонизированных со стандартами МЭК и IEC.

Представленный материал полезен для обучающихся и способствует повышению уровня подготовки в рамках требуемых профессиональных компетенций в области возобновляемой энергетики и систем накопления.

\section{Список литературы}

1. Рынок систем накопления электроэнергии в России: Потенциал развития. Экспертно-аналитический доклад. / Под ред. Ю. Удальцова и Д. Холкина. РОСНАНО. Москва, 2018. - 70 с. // [Электронный ресурс]. - Режим доступа: en.rusnano.com. -Дата обращения 10.12. 2020.

2. Системы накопления электрической энергии (СНЭЭ). Термины и определения: ГОСТ Р 58092.1-2018 / введ. 2019-03-01. - Москва: Стандартинформ, 2018. - 54 с.

3. Системы накопления электрической энергии (СНЭЭ). Параметры установок и методы испытаний. Общее описание: ГОСТ Р 58092.2.1-2020 (МЭК 62933-2-1:2017) / введ. 2020-11-01. Москва: Стандартинформ, 2020. 37 с.

4. Системы накопления электрической энергии (СНЭЭ). Проектирование и оценка рабочих параметров. Общие требования: ГОСТ Р 58092.3.1-2020 (IEC TS 62933-3-1:2018)/ введ. 2020-11-01. - Москва: Стандартинформ, 2020. - 56 с.

5. Андрианова, Л.П. Терминология для классификации проектирования и установки систем накопления электроэнергии в интеллектуальной энергетике / Л.П. Андрианова, 3.Х. Павлова, М.И. Хакимьянов, Р.Т. Хазиева // Новая наукаНовые возможности: сборник статей Междунродного научноисследовательского конкурса (1 ноября 2020 г.). - Петрозаводск: МЦНП «Новая наука», 2020. - 73 с. - C.24-36.

6. Андрианова, Л.П. Понятийный аппарат для установления требований к системам накопления электроэнергии, взаимодействующими с электрической сетью / Л.П. Андрианова, 3.Х. Павлова, М.И. Хакимьянов, Р.Т. Хазиева // Инновационное развитие науки: Фундаментальные и прикладные проблемы: монография. - Петрозаводск: МЦНП «Новая наука», 2020. - 92 с. - С.78-93.

() Л.П. Андрианова, 3.Х. Павлова, Л.А. Рябишина, М.И. Хакимьянов, Р.Т. Хазиева, 2020 